\title{
COVID-19 infection in NMO/SD patients: a French survey
}

\author{
Sinéad Zeidan ${ }^{1} \cdot$ Elisabeth Maillart ${ }^{1} \cdot$ Céline Louapre $^{1,2} \cdot$ Thomas Roux $^{1} \cdot$ Catherine Lubetzki $^{1,2} \cdot$ Caroline Papeix $^{1} \mathbb{C}$
}

Received: 30 June 2020 / Revised: 21 July 2020 / Accepted: 23 July 2020 / Published online: 12 September 2020

(c) Springer-Verlag GmbH Germany, part of Springer Nature 2020

Keyword NMOSD · COVID-19 · Immunosuppressants · AQP4

Dear Sirs,

COVID-19 pandemic due to SARS-CoV2 virus is ongoing, with a fatality rate around $5.4 \%$ [1]. Neuromyelitis optica spectrum disorders (NMO/SD) is a rare disabling disease requiring immunosuppressive therapy. The risk of severe COVID-19 remains unknown in NMO/SD. The aim of this case-series study is to describe the prevalence and characteristics of COVID-19 in NMO/SD patients.

We conducted a monocentric retrospective caseseries study of NMO/SD patients with highly suspected or proven COVID-19. They fulfilled at least one of the three NMO diagnostic criteria sets [2-4] and were registered in the NOMADMUS cohort (gathering data from French expert NMO/SD centers). Among $117 \mathrm{NMO} /$ SD patients followed in the department of neurology of Pitié Salpêtrière Hospital in Paris, 75 were questioned by phone about COVID-19 infection between 05/05/2020 and 06/15/2020 (33 were lost to follow-up, 9 were unreachable by phone). We collected demographics, neurological history, comorbidities, COVID-19 characteristics and outcome. COVID-19 diagnosis was retained on $\geq 1$ of the following criteria was fulfilled: (1) positive SARS-CoV2 PCR (naso-pharyngeal swab) or serology (IgG); (2) typical thoracic ground glass opacities on CT scan; (3) acute anosmia/ageusia of sudden onset in the absence of rhinitis or nasal obstruction; (4) typical triad symptoms (cough, fever, asthenia) in epidemic zone of COVID-19. The study received approval from the ethic committee of Sorbonne University (\#CER-2020-19).

Caroline Papeix

caroline.papeix@aphp.fr

1 Department of Neurology, Pitié Salpêtrière Hospital, APHP, Sorbonne University, 75013 Paris, France

2 Institut du Cerveau et de la Moelle épinière, ICM, CIC Neuroscience, Paris, France
Demographical and clinical characteristics of the 75 $\mathrm{NMO} / \mathrm{SD}$ questioned by phone are summarized in Table 1 . During the lockdown in France (03/17/2020-05/11/2020), neurologists were available at hospital and by phone/video consults. NMO/SD treatments were maintained, including in-hospital infusions. Patients mostly stopped $100 \%$ $(n=44 ; 58.7 \%)$ or $>50 \%$ of their outings $(n=29 ; 38.7 \%)$; two patients $(2.7 \%)$ continued to attend their workplace. Nine patients (12.0\%) reported an interaction with a symptomatic person.

Five patients ( $6.7 \%$ of the whole cohort) fulfilled the diagnosis of COVID-19 (Table 2). Their last biological analysis before COVID-19 [median (Q1-Q3) delay: 45 (10-60) days] found: grade 1 lymphopenia $(n=3 / 5)$ or normal lymphocyte count $(n=1 / 5)$, and normal neutrophilic counts $(n=4 / 5$, missing data for one patient). The two patients receiving anti-CD20 treatment had a B-lymphocyte depletion. No patient had a severe infection or neurological state worsening. One patient treated with anti-CD20 and weekly plasmapheresis was hospitalized for closed monitoring he did not require oxygenotherapy, had a normal thoracic CT scan, and plasmapheresis was resumed three weeks later.

In this systematic survey of $75 \mathrm{NMO} / \mathrm{SD}$ patients, $6.7 \%$ had a highly suspected or confirmed COVID-19, which is consistent with the estimated seroprevalence in Ilede-France [5]. This rate is slightly higher than the 3.8\% prevalence reported in an Iranian study [6], while a large survey in China described a very low risk of COVID-19: 2 confirmed COVID-19 pneumonia out of 3060 NMO/SD patients [7]. Despite ongoing immunosuppressive therapy, no severe infection was encountered in our study. It may be related to the young age and low rate of comorbidity in the infected patients. Some authors reported a favorable outcome of COVID-19 in anti-CD20-treated patients [8,9], possibly due to its minor impact on T-cell counts [10]. A 
Table 1 Characteristics of the NMO/SD cohort $(n=75)$

\begin{tabular}{ll}
\hline Female, $n(\%)$ & $59(78.7 \%)$ \\
Age, mean $( \pm \mathrm{SD})$ & $43.9( \pm 14.1)$ \\
Serology, $n(\%)$ & \\
Positive AQP4-IgG & $-46(61.3 \%)$ \\
Positive MOG-IgG & $-23(30.7 \%)$ \\
Negative AQP4- and MOG-IgG & $-6(8.0 \%)$ \\
EDSS, median [Q1-Q3] & $4.5(2.0-7.0)$ \\
Disease duration, median [Q1-Q3] & $6.0(3.0-11.5)$ \\
Immunosuppressive treatment, $n(\%):$ & \\
Azathioprine & $-23(30.7 \%)$ \\
Mycophenolate mofetil & $-19(25.3 \%)$ \\
Anti-CD20 therapy & $-20(26.7 \%)$ \\
Plasmapheresis & $-4(5.3 \%$ \\
Mitoxantrone & $-1(1.3 \%)$ \\
Intravenous immunoglobulin & $-1(1.3 \%)$ \\
Add-on corticotherapy, $n(\%)$ & $9(12.0 \%)$ \\
\hline
\end{tabular}

beneficial effect on the inflammatory response to SARSCoV2 remains to be confirmed [11], but other immunosuppressive treatments are studied for this purpose [12]. As we observed in 2 patients, anti-CD20 treatment may prevent the development of antibodies targeting SARSCoV2 [8].

Our study has limitations: the sample size is small and COVID-19 tests were poorly available during the outbreak of SARS-CoV2 in France: hence, they were not performed in asymptomatic patients. However, this systematic survey of a French NMO/SD center has resulted in the detection of mild cases of COVID-19 among this immunosuppressed population, which probably would not have been reported otherwise. It highlights a similar prevalence to the local population and a benign course of COVID-19, without any case of flare-up of the neurologic disease. Consistently with a French cohort study on MS patients [13], this survey is reassuring about the risk of severe COVID-19 in NMO/ SD-immunosuppressed patients and supports the maintenance of immunosuppressive treatments for NMO/SD during COVID-19 pandemic. Nevertheless, social distancing should still be maintained in case of another outbreak of SARS-CoV2.

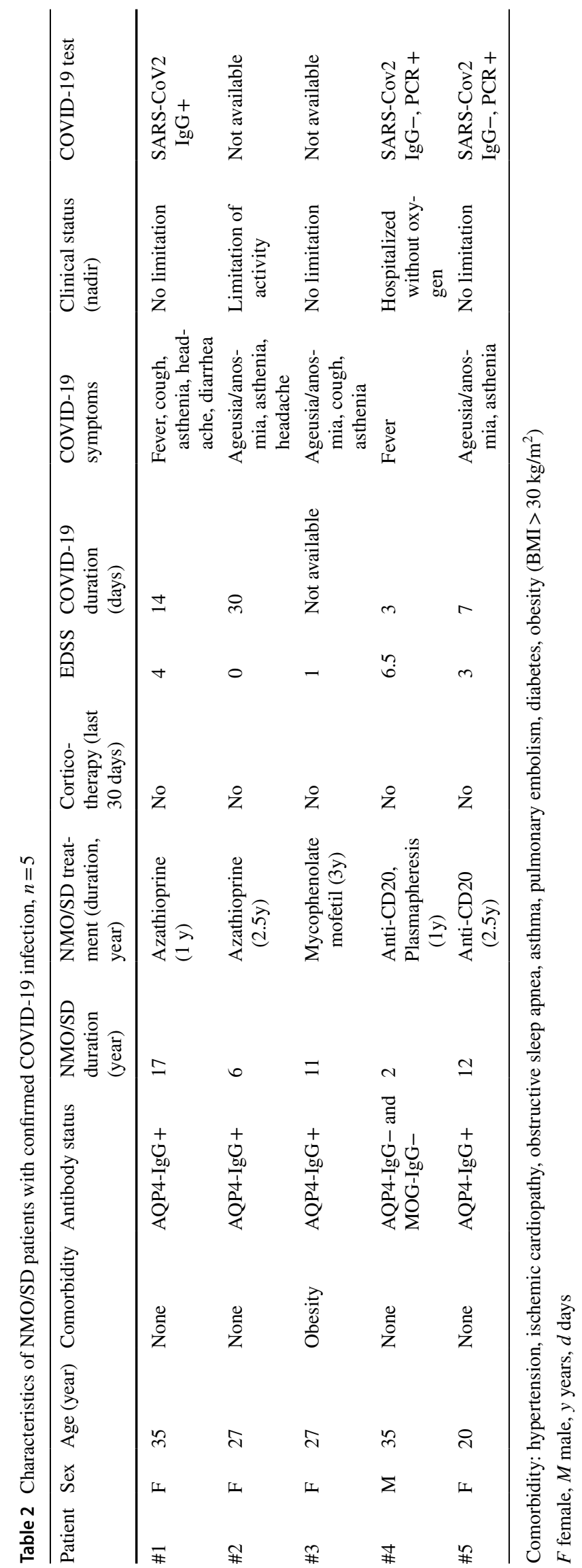




\section{Compliance with ethical standards}

Conflicts of interest On behalf of all authors, the corresponding author states that there is no conflict of interest.

\section{References}

1. Tu Y-F, Chien C-S, Yarmishyn AA et al (2020) A Review of SARS-CoV-2 and the ongoing clinical trials. Int J Mol Sci 21:2657. https://doi.org/10.3390/ijms21072657

2. Wingerchuk DM, Hogancamp WF, O'Brien PC, Weinshenker BG (1999) The clinical course of neuromyelitis optica (Devic's syndrome). Neurology 53:1107-1114. https://doi.org/10.1212/ wnl.53.5.1107

3. Wingerchuk DM, Lennon VA, Pittock SJ et al (2006) Revised diagnostic criteria for neuromyelitis optica. Neurology 66:14851489. https://doi.org/10.1212/01.wnl.0000216139.44259.74

4. Wingerchuk DM, Banwell B, Bennett JL et al (2015) International consensus diagnostic criteria for neuromyelitis optica spectrum disorders. Neurology 85:177-189. https://doi.org/10.1212/ WNL.0000000000001729

5. Salje H, Tran Kiem C, Lefrancq N et al (2020) Estimating the burden of SARS-CoV-2 in France. Science. https://doi.org/10.1126/ science.abc3517

6. Sahraian MA, Azimi A, Navardi S et al (2020) Evaluation of COVID-19 infection in patients with Neuromyelitis optica spectrum disorder (NMOSD): a report from Iran. Mult Scler Relat Disord 44:102245. https://doi.org/10.1016/j.msard.2020.102245
7. Fan M, Qiu W, Bu B et al (2020) Risk of COVID-19 infection in MS and neuromyelitis optica spectrum disorders. Neurol Neuroimmunol Neuroinflamm. https://doi.org/10.1212/NXI.00000 00000000787

8. Meca-Lallana V, Aguirre C, Río B, null et al (2020) COVID-19 in 7 multiple sclerosis patients in treatment with ANTI-CD20 therapies. Mult Scler Relat Disord 44:102306. https://doi. org/10.1016/j.msard.2020.102306

9. Montero-Escribano P, Matías-Guiu J, Gómez-Iglesias P et al (2020) Anti-CD20 and COVID-19 in multiple sclerosis and related disorders: a case series of 60 patients from Madrid. Spain Mult Scler Relat Disord 42:102185. https://doi.org/10.1016/j. msard.2020.102185

10. Giovannoni G, Hawkes C, Lechner-Scott J et al (2020) The COVID-19 pandemic and the use of MS disease-modifying therapies. Mult Scler Relat Disord 39:102073. https://doi. org/10.1016/j.msard.2020.102073

11. Novi G, Mikulska M, Briano F et al (2020) COVID-19 in a MS patient treated with ocrelizumab: does immunosuppression have a protective role? Mult Scler Relat Disord 42:102120. https://doi. org/10.1016/j.msard.2020.102120

12. Toniati P, Piva S, Cattalini M et al (2020) Tocilizumab for the treatment of severe COVID-19 pneumonia with hyperinflammatory syndrome and acute respiratory failure: a single center study of 100 patients in Brescia. Italy Autoimmun Rev 19:102568. https ://doi.org/10.1016/j.autrev.2020.102568

13. Louapre C, Collongues N, Stankoff B et al (2020) Clinical characteristics and outcomes in patients with coronavirus disease 2019 and multiple sclerosis. JAMA Neurol. https://doi.org/10.1001/ jamaneurol.2020.2581 